\title{
GPU-Based Lighting and Shadowing of Complex Natural Scenes
}

\author{
Florent Cohen* \\ GRAVIR/IMAG-INRIA \\ Philippe Decaudin* \\ Fabrice Neyret* \\ www-imagis.imag.fr/Publications/2004/CDN04/
}

\section{Purpose and motivations}

Rendering realistic natural scenes in real-time is a challenging topic in the field of computer graphics. More specifically, we want to render scenes that contain complex shapes bounded in a layer above a surface. Landscapes (e.g., terrain, vegetation) are examples of such scenes. Lightings and shadows are essential elements toward realism because they help understanding shapes. However:

- Using polygonal meshes and classical shadowing algorithms are too costly in terms of computation for complex scenes;

- Adapted algorithms for dedicated representations such heightfields are efficient, but these representations are too limited for our needs (e.g. forest);

- Although more powerful representations like surfels or texels exist, algorithms for quality and efficient lighting and shadowing are not available yet.

Thus, our goal is to propose lighting and shadowing techniques adapted to such representations as well as the GPU.

\section{Lighting}

In order to highlight complex scenes, per-pixel lighting is required. In order to compute it accurately, we should rely on the normals distribution of the elements which project on each pixel. This data can be obtained from the shape representation (e.g., see [Cohen et al. 1998] for meshes). Note that bump mapping techniques correspond to the brutal undersampling of this distribution (1 normal per pixel). Since it is not properly filtered strong aliasing can appear. [Fournier 1992] gives some ideas for representing and filtering the normals distribution. We propose to rely on [Lafortune et al. 1997] shading model to represent and filter the normal distributions.

The filtering will be pre-computed and stored in textures, allowing the GPU to process the computation quickly.

\section{Shadowing}

Processing the shadows on as per object basis is often impractical since the amount of objects is very large. Instead, we extend the horizon mapping techniques [Max 1988; Sloan and Cohen 2000] in such a way that it is adapted to non-heightfield scenes: we precompute several horizon maps at differents heights above the surface (see below). These maps are then interpolated for each given fragment within the volumeric layer. Thus, shadows can be rendered at low cost compared to the visual complexity.

\section{Application: Forest scenes}

In order to test and validate our approach, we implemented our techniques based on volume encoded forest scenes [Decaudin and Neyret 2004], in which precomputed lighting and shadows were used. We pre-computed a 3D "texcell" containing the normals of each forest scene element.

For lighting, we currently calculate a simple dot product between normals and light vector. The next step will be to encode the Lafortune model and to evaluate it in a fragment program.

For shadowing, we pre-computed two horizon maps at the top and bottom of the forest, and let the ordinary texture management interpolate them for each fragment.

Results are visually promising, but some problems still remained: some major optimizations are still pending, and we are currently working on implementing Lafortune normals filtering.

We had achieved 5 frames per second on each pictured scene on a Pentium IV 2 GHz with a GeForce FX5800.
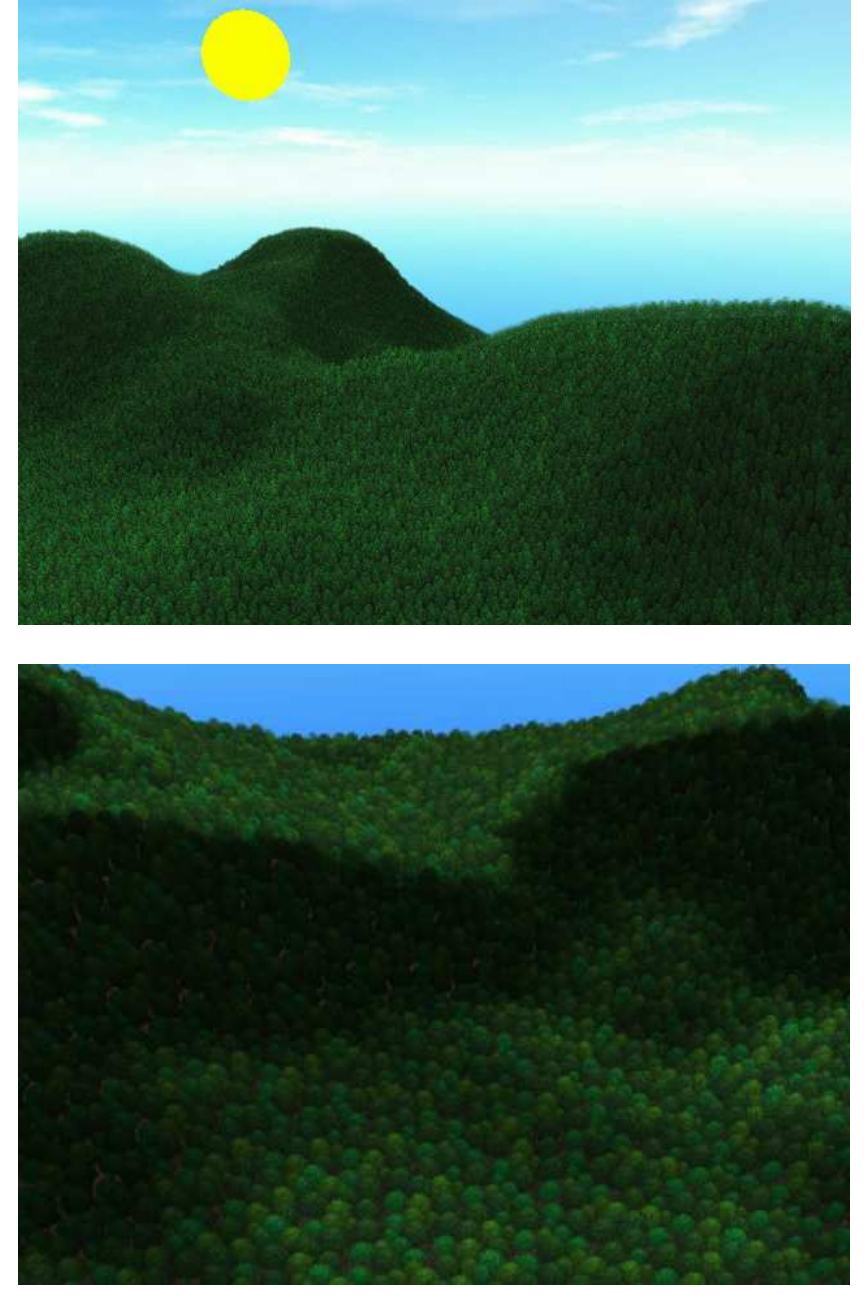

\section{References}

Cohen, J., Olano, M., And Manocha, D. 1998. Appearanceperserving simplification. In Proc. of the 25th annual conference on Computer graphics and interactive techniques, 115-122.

Decaudin, P., AND NeYRet, F. 2004. Rendering forest scenes in real-time. In Eurographics Symposium on Rendering 2004.

FoURNIER, A. 1992. Normal distribution functions and multiple surfaces. In Graphics Interface '92 Workshop on Local Illumination, 45-52.

Lafortune, E. P. F., Foo, S.-C., Torrance, K. E., And GREENBERG, D. P. 1997. Non-linear approximation of reflectance functions. In Proc. of SIGGRAPH 97.

MAX, N. L. 1988. Horizon mapping: shadows for bump-mapped surfaces. The Visual Computer 4, 2 (July), 109-117.

SloAn, P., AND CoHen, M. F. 2000. Hardware accelerated horizon mapping. In Rendering Techniques 2000: 11th Eurographics Workshop on Rendering, 281-286.

\footnotetext{
*e-mails: firstname.name@imag.fr
} 